\title{
Pathological Investigation of Vertebral Tumor Metastasis from Unknown Primaries - a Systematic Analysis
}

\author{
Yan Zhang ${ }^{\star}$, Feng Cai ${ }^{\circledR}$, Liang Liu, Xiao-Dong Liu*
}

\begin{abstract}
Background: This systematic analysis was conducted to investigate pathological diagnosis of vertebral tumor metastasis with unknown primaries. Methods: Clinical studies conducted to pathologically investigate vertebral tumor metastasis were identified using a predefined search strategy. Pooled diagnosis (PD) of each pathological confirmation was calculated. Results: For vertebral tumor metastasis, 5 clinical studies which included 762 patients were considered eligible for inclusion. Systematic analysis suggested that, for all patients with vertebral tumor metastasis, dominant PD was pathologically confirmed with lung cancer in $21.7 \%(165 / 762)$, with breast cancer in $26.6 \%(203 / 762)$ and with prostate cancer in $19.2 \%(146 / 762)$. Other diagnosis that could be confirmed included lymphoma, multiple myeloma, renal cancer, for example, in this cohort of patients. Conclusions: This systemic analysis suggested that breast, lung and prostate lesions could be the most common pathological types of cancer for vertebral tumor metastasis formunknown primaries, and other common diagnoses could include lymphoma, multiple myeloma, renal cancer.
\end{abstract}

Keywords: Pathological diagnosis - vertebral tumour metastasis

Asian Pac J Cancer Prev, 16 (3), 1047-1049

\section{Introduction}

Vertebral tumor metastasis is common, 60 to $70 \%$ of patients with systemic cancers have vertebral tumor metastasis (Maillefert et al., 2000; Destombe et al., 2007). And it is estimated that on this occasion the primary tumor is unknown at the initial diagnosis in 0.5 to $7 \%$ of these patients (Abbruzzese et al., 1994; Daugaard et al., 1994). Previous report suggested that cancers of unknown primary origin increased substantially. 9 In a postmortem study of such patients, the lung and the large intestine were the most frequent sites for primary tumors (Al-Brahim et al., 2005).Open biopsies of vertebral tumors carry a potential risk of complications (Mankin et al., 1982; Mankin et al., 1996). So, conventional techniques for evaluation of primary tumor foci (thyroid, colon, prostate, lung) are time-consuming, risky and expensive.

The purpose of this systemic analysis was conducted to investigate possible pathological diagnosis of vertebral tumor metastasis that is unknown primaries.

\section{Materials and Methods}

\section{Search strategy}

We searched PUBMED, by using the following search term: (pathological diagnosis) and (vertebral tumor metastasis). All clinical studies evaluating the outcome of possible pathological diagnosis on vertebral tumor metastasis and published in English prior to December 2014 were identified. If samples of two studies overlap, only the newest one was included. Additional articles were obtained from references within the articles identified by the electronic search. We did not consider meeting abstracts or unpublished reports.

\section{Inclusion and exclusion criteria}

We reviewed abstracts of all citations and retrieved studies. The following criteria were used to include published studies: (1) clinical studies, containing key words pathological diagnosis and vertebral tumor metastasis; (2) The study was performed in accordance with the Helsinki Declaration (1964, amended in 1975 and 1983) of the World Medical Association. Eligibility criteria included vertebral tumor metastasis with the primary tumor is unknown at the initial diagnosis, the presence of at least one bidimensionally measurable lesion. Studies were excluded if one of the following existed: (a) duplicate data; (b) no sufficient data were reported.

\section{Data collection and analysis}

Selection of trials and data extraction: The titles and abstracts of publications identified according to the above search strategy were assessed independently for inclusion by two authors; the full text was selected for further assessment if the abstract suggests relevance. 
Disagreement was resolved by discussion. Data was extracted by independent authors. The following recorded data were extracted: author, publication data, and country of the first or corresponding author, the number of patients. Outcome measures presented in at least 3 studies were extracted for combined analysis.

\section{Results}

We searched PUBMED, by using the following search term: (pathological diagnosis) and (vertebral tumor metastasis). There were 928 papers relevant to the search words by the end of December 2014. Via steps of screening the title and reading the abstract, 5 studies were identified (Roth et al., 2004; Chaichana et al., 2009; Husband et al., 2001; Lu et al., 2005; Helweg-Larsen et al., 2000). These studies had been carried out in China, and the USA. The following outcomes were presented in at least all studies and extracted for combined analysis: the relationship between pathological diagnosis and vertebral tumor metastasis regarding clinical manifestation, or spinal cord or nerve root compression as well as the clinical severity of the compression. Characteristics of studies included in the analysis are presented as these outcomes: primary cancer diagnoses were reported as follows: breast, $n=23$; lung, $\mathrm{n}=7$; colon, $\mathrm{n}=3$; prostate, $\mathrm{n}=5$; lymphoma, $\mathrm{n}=6$; multiple myeloma, $n=5$; renal, $n=4$; other, $n=10$; and unknown, $n=9$ of Roth et al.; primary cancer diagnoses among the 162 included patients were various, reported as follows: lung $(n=26,16 \%)$, breast $(n=26,16 \%)$, prostate $(n=20,12 \%)$, renal $(n=21,13 \%)$ and hematopoietic $(n=28,17 \%)$. of Chaichana et al.; The primary cancer diagnoses among the 280 included patients were various and reported as follows: breast 65 , prostate 57 , bronchus 72 , hematological 23 , urinary tract 21 , gastrointestinal tract 13 , unknown primary 12 and other 17 patients of Husband et al; primary cancer diagnoses were reported as breast $(n=33 ; 24 \%)$, lung $(n=33 ; 24 \%)$, prostate $(n=21$; $15 \%)$, non-Hodgkin's lymphoma $(\mathrm{n}=8 ; 6 \%)$, multiple myeloma $(n=6 ; 4 \%)$ and others $(n=35,26 \%)$ of Lu et al. and primary cancer diagnoses were breast carcinoma in 56 patients $(37 \%)$, prostatic carcinoma in $43(28 \%)$, NSCLC in $18(12 \%)$, SCLC in nine $(6 \%)$, and other solid tumors in $27(17 \%)$ patients of Helweg-Larsen et al. Totally, 762 patients from five clinical studies were included, and the primary cancer diagnoses were breast carcinoma in patients 203(26.6\%), lung cancer in $165(21.7 \%)$,prostatic carcinoma in $146(19.2 \%)$.

\section{Discussion}

The spine is a common site for bone metastasis. When the primary site of a metastatic cancer is known, diagnostic methods including plain radiography, myelography, magnetic resonance imaging, computerised tomography, radionuclide bone scanning (scintigraphy with technetium99m-labelled diphosphonates), single-photon emission CT and positron emission tomography should be firstly scheduled.And in this setting, pathological diagnosis could be made according to the histological type of the corresponding site (Zhao et al., 2014). If the primary site is unknown, biopsy should be considered for pathological diagnosis. Usually, small-diameter needles are used first, as largebore needles are associated with higher rates of complication (Nourbakhsh et al., 2008; Yaffe et al., 2003). Complication rates of transpedicular biopsy are 0 to $26 \%$; pulmonary, neurologic, and infectious complications are considered the most common (Laredo et al.1986; Fyfe et al.1983; Bender et al., 1986).Percutaneous biopsy of the vertebra can be performed on an outpatient basis under local anaesthesia. The necessary surgical equipment is cheap and the procedure does not require extra assistance. It is a cost-effective diagnostic tool for evaluating patients with unknown primary tumors. In 15 to $25 \%$ of such cases, the primary site cannot be identified even at necropsy (Neumann et al., 2002). wFluorodeoxyglucose positron emission tomography and computed tomography is recommended to diagnose, stage, and restage a variety of cancers (Czernin et al., 2002), especially for these patients with vertebral tumor metastasis and the primary site cannot be identified (Rosenbaum et al., 2006; Dong et al., 2006; Chang et al., 2006). Advanced diagnostic methods in pathology (immunohistochemistry and immunofluorescence) enable accurate diagnosis of the origin of primary tumors by biopsy of the metastatic when the primary site of a metastatic cancer is known, biopsy is usually not required. The purpose of pathological diagnosis includes the following points: (1) to inform the choice about potential preemptive intervention(s) so as to avoid or delay complication and more radical surgical intervention; (2) to bring forward radical interventions before patient health deteriorates too far; and (3) to categorise patients into those more or less suitable for earlier or later radical intervention. However, there is uncertainty about the effectiveness of these diagnostic techniques. Therefore, investigation on clinical manifestation of spine metastasis of unknown primary is very important.

There were several studies in which clinical manifestation of spine metastasis from different primary tumor types was investigated. Two studies (Shah et al., 2003; Chaichana et al., 2009) found that primary tumor type was a risk factor for vertebral collapse. Similarly, three studies (Klekamp et al., 1998; Helweg-Larsen et al., 2000) found that primary tumor type was a risk factor for disease recurrence, and Klekamp et al. found that patient health status was also influential. Three studies (Taneichi et al., 1997; Roth et al., 2004; Rose et al., 2009) all found that degree of tumor occupancy of the vertebral body was predictive for fracture. Thus, it could be demonstrated that clinical manifestation appears to vary with pathological diagnosis of the primary tumor, and the length of time from primary diagnosis to study entry, will influence the results of any analysis of prognostic factors. This leads to considerable difficulty interpreting the results from previous studies. Potential confounding effects of primary tumour type, age and treatment with bisphosphonates or other interventions should have been considered when attempting to identify clinical variables.

Our current retrospectivelly conducted studies that were focusing on analyzing characteristics of vertebral tumor metastasis, 5 clinical studies which including 762 patients were considered eligible for inclusion. And our 
analysis suggested that, for all patients, dominant PD that was pathologically confirmed with lung cancer in $21.7 \%$ $(165 / 762)$, with breast cancer in $26.6 \%(203 / 762)$ and with prostate cancer in $19.2 \%(146 / 762)$ of patients with vertebral tumor metastasis. Other diagnosis that could be confirmed included lymphoma, multiple myeloma, renal cancer, etc in this cohort of patients.

In conclusion, our current systemic analysis suggests that breast, lung and prostate could be the most common pathological type on vertebral tumor metastasis that is unknown primaries, and the diagnosis also could include lymphoma, multiple myeloma, renal cancer, etc.

\section{Acknowledgements}

This work was supported by National Natural Science Foundation of China (NSFC, No.81372015) and Shanghai Municipal Health Bureau Project (No. 2013214).

\section{References}

Abbruzzese JL, Abbruzzese MC, Hess KR, et al (1994). Unknown primary carcinoma: natural history and prognostic factors in 657 consecutive patients. J Clin Oncol, 12, 127280.

Al-Brahim N, Ross C, Carter B, et al (2005). The value of postmortem examination in cases of metastasis of unknown origin-20-year retrospective data from a tertiary care center. Ann Diagn Pathol, 9, 77-80.

Bender CE, Berquist TH, Wold LE (1986). Imaging-assisted percutaneous biopsy of the thoracic spine. Mayo Clin Proc, 61, 942-50.

Chaichana KL, Pendleton C, Wolinsky JP, et al (2009). Vertebral compression fractures in patients presenting with metastatic epidural spinal cord compression. Neurosurgery, 65, 267-74.

Chang JM, Lee HJ, Goo JM, et al (2006). False positive and false negative FDG-PET scans in various thoracic diseases. Korean J Radiol, 7, 57-69.

Czernin J (2002). Clinical applications of FDG-PET in oncology. Acta Med Austriaca, 29, 162 -70.

Daugaard G (1994). Unknown primary tumours. Cancer Treat Rev, 20, 119-47.

Destombe C, Botton E, Le Gal G, et al (2007). Investigations for bone metastasis from an unknown primary. Joint Bone Spine, 74, 85-17.

Dong MJ, Lin XT, Zhao J, et al (2006). Malignant tumor with false negative 18F-FDG PET image. Zhonghua Zhong Liu Za Zhi, 28, 713-7.

Fyfe IS, Henry AP, Mulholland RC (1983). Closed vertebral biopsy. J Bone Joint Surg Br, 65, 140-3.

Helweg-Larsen S, Sorensen PS, Kreiner S (2000). Prognostic factors in metastatic spinal cord compression: a prospective study using multivariate analysis of variables influencing survival and gait function in 153 patients. Int J Radiat Oncol Biol Phys, 46 1163-9.

Husband DJ, Grant KA, Romaniuk CS (2001). MRI in the diagnosis and treatment of suspected malignant spinal cord compression. Br J Radiol, 74, 15-23.

Klekamp J, Samii H (1998). Surgical results for spinal metastases. Acta Neurochir, 140, 957-67.

Laredo JD, Bard M (1986). Thoracic spine: percutaneous trephine biopsy. Radiology, 160, 485-9.

Levack P, Graham J, Collie D, et al (2002). Don't wait for a sensory level-listen to the symptoms: a prospective audit of the delays in diagnosis of malignant cord compression.
Clin Oncol ( $R$ Coll Radiol), 14, 472-80.

Lu C, Gonzalez RG, Jolesz FA, et al (2005). Suspected spinal cord compression in cancer patients: a multidisciplinary risk assessment. J Support Oncol, 3, 305-12.

Maillefert JF, Tavernier C, Tebib J (2000). Determining the site of the primary cancer in patients with skeletal metastasis of unknown origin: a retrospective study. Cancer, 88, 1759-61.

Mankin HJ, Lange TA, Spanier SS (1982). The hazards of biopsy in patients with malignant primary bone and soft-tissue tumors. J Bone Joint Surg Am, 64, 1121-7.

Mankin HJ, Mankin CJ, Simon MA (1996). The hazards of the biopsy, revisited. Members of the Musculoskeletal Tumor Society. J Bone Joint Surg Am, 78, 656-63.

Minardi AJ Jr, Sittig KM, Zibari GB, et al (1998). Colorectal cancer in the young patient. Am Surg, 64, 849-53.

Muir C (1995). Cancer of unknown primary site. Cancer, $\mathbf{7 5}$, 353-6.

Neumann KH, Nystrom JS (1982). Metastatic cancer of unknown origin: nonsquamous cell type. Semin Oncol, 9, 427-34.

Nourbakhsh A, Grady JJ, Garges KJ (2008). Percutaneous spine biopsy: a meta-analysis. J Bone Joint Surg Am, 90, 1722-5.

Rose PS, Laufer I, Boland PJ, et al (2009). Risk of fracture after single fraction image-guided intensity-modulated radiation therapy to spinal metastases. J Clin Oncol, 27, 5075-9.

Rosenbaum SJ, Lind T, Antoch G, et al (2006). False-positive FDG PET uptake-the role of PET/CT. Eur Radiol, 16, 1054-65.

Roth SE, Mousavi P, Finkelstein J, et al (2004). Metastatic burst fracture risk prediction using biomechanically based equations. Clin Orthop Relat Res, 83-90.

Shah AN, Pietrobon R, Richardson WJ, et al (2003). Patterns of tumor spread and risk of fracture and epidural impingement in metastatic vertebrae. J Spinal Disord Tech, 16, 83-9.

Talcott JA, Stomper PC, Drislane FW, et al (1999). Assessing suspected spinal cord compression: a multidisciplinary outcomes analysis of 342 episodes. Support Care Cancer, 7,31-8.

Taneichi H, Kaneda K, Takeda N, et al (1997). Risk factors and probability of vertebral body collapse in metastases of the thoracic and lumbar spine. Spine, 22, 239-45.

Yaffe D, Greenberg G, Leitner J, et al (2003). CT-guided percutaneous biopsy of thoracic and lumbar spine: a new coaxial technique. AJNR Am J Neuroradiol, 24, 2111-3.

Zhao CL, Qian GQ, Chen XY, et al (2014). Retrograde analysis of clinical characteristics of bone metastasis in 1,031 cases of preliminarily diagnosed nasopharyngeal carcinoma. Asian Pac J Cancer Prev, 15, 3785-8. 